\title{
Robust Three-dimensional (3D) Expansion of Bovine Intestinal Stem Cells: A Model for Replacement of Animal Experiments
}

Bo Ram Lee ( $\nabla$ mir88@korea.kr)

National Institute of Animal Science https://orcid.org/0000-0002-0537-6205

Hyeon Yang

National Institute of Animal Science

Sang In Lee

Kyungpook National University

Inamul Haq

National Institute of Animal Science

Sun A Ock

National Institute of Animal Science

Hayeon Wi

National Institute of Animal Science

Hwi Cheul Lee

National Institute of Animal Science

Poongyeon Lee

National Institute of Animal Science

Jae Goo Ryu

National Institute of Animal Science

\section{Research}

Keywords: Bovine, Intestinal Stem Cells, Organoid, Characterization, Gene expression

Posted Date: January 29th, 2021

DOI: https://doi.org/10.21203/rs.3.rs-164747/v1

License: (c) (i) This work is licensed under a Creative Commons Attribution 4.0 International License.

Read Full License 


\section{Abstract}

Background Intestinal organoids offer great promise for disease modelling based host-pathogen interactions and nutritional research for feed efficiency measurement in livestock as well as regenerative medicine for therapeutic purposes. However, very limited studies are available on the functional characterization and three-dimensional (3D) expansion of adult stem cells in livestock species compared to mammals. Therefore, we characterized intestinal stem cells derived from small intestine in adult bovine and cultivated intestinal organoids under in vitro 3D culture system.

Results In this study, we successfully established intestinal organoids in bovine. Intestinal organoids were long-term cultivated over several passages of culture without loss of the recapitulating capacity of crypts and they had the specific expression of several specific markers involved in intestinal stem cells, intestinal epithelium and nutrient absorption. In addition, they showed the key functionality with regard to a high permeability for compounds of up to FITC-dextran $4 \mathrm{kDa}$, while FITC-dextran $40 \mathrm{kDa}$ failed to enter the organoid lumen. Furthermore, the genetic properties of intestinal organoids were highly similar to those of in vivo based on QuantSeq 3' mRNA-Seq. data.

Conclusions Collectively, these results provide a reliable method for efficient isolation of intestinal crypts from small intestine and robust 3D expansion of intestinal stem cells in adult bovine and demonstrate the in vitro 3D organoids mimics the in vivo tissue topology and functionality. Finally, intestinal organoids are potential alternatives to in vivo system and will facilitate the practical use of a model to replace animal experiments in the fields of animal biotechnology for various purposes.

\section{Background}

The ability to recapitulate stem cells' self-organizing potential, creating three-dimensional (3D) structures of stem cells, has revolutionized various fields in regenerative medicine and the fundamental study of biological processes such as growth and differentiation [1, 2]. In particular, these organoid-based systems hold great promise for further potential use in determining disease modelling based host-pathogen interactions and nutritional research for feed efficiency measurements to improve productivity in the fields of animal biotechnology [2, 3]. Recently, significant efforts have been made to establish a physiologically relevant in vitro system based on 3D organoids, including the small intestine and liver, in animals for practical applications, resulting in the reduction and replacement of animal experiments [4-6]. However, very limited studies are available on the functional characterization and 3D expansion of adult stem cells isolated from livestock compared to any other animal.

Organoids have been successfully developed from two sources of stem cells, organ-restricted adult stem cells (ASCs) from various sets of organs, such as the intestine, kidney, lung, liver, pancreas, and brain, and pluripotent stem cells, including embryonic stem cells and induced pluripotent stem cells [7]. Since the first report in 2009 [8], intestinal organoids, such as the mini-gut, have evolved as a potential alternative to in vivo models for the use of various purposes [9]. They are capable of closely replicating the structure 
and cellular composition of a functional native intestinal epithelium, including intestinal cell types, e.g., enterocytes, goblet cells and paneth cells $[8,10]$. Consequently, in vitro 3D organoid systems are now used as alternative research tools because they serve the same purpose as in vivo systems.

Cattle are an economically important domestic animal species for the production of milk and meat worldwide [4]. However, cattle, as experimental animals, are highly expensive and labour intensive, particularly when sampling and separating several different tissues from slaughter [11]. Moreover, in vitro 2D culture system such as immortalized [12] and cloned [13] intestinal epithelial cell lines have the current limitation due to lacking of physiological relevance and the cellular diversity composed of the intestinal epithelium [4]. Therefore, the development of a reliable method for robust 3D culture system derived organ-restricted ASCs from various sets of organs in livestock is urgently necessary.

In the current study, we successfully established bovine intestinal organoids representing typical cryptvillus structure and intestinal epithelium and characterized the expression levels of several specific markers in reference to intestinal stem cells and nutrient uptake using QuantSeq 3' RNA-Seq. data and immunocytochemistry. Furthermore, we investigated epithelial barrier function using a FITC-dextran permeability assay.

\section{Method}

\section{Experimental designs, animals and animal care}

This study was designed with the aim of investigating adult derived-intestinal stem cells and subsequent derivation and cultivation of intestinal organoids in bovine. Hanwoo cattle (Bos Taurus coreanae) $(>24$ months old) were used experimentally in this study after receiving approval from the Institutional Animal Care and Use Committee (IACUC) of the National Institute of Animal Science (NIAS-2019-366), Korea. All procedures, including sample collection and handling, followed the standard operating protocols of the Animal Biotechnology Division at the National Institute of Animal Science, Korea.

\section{Isolation of intestinal crypts and three-dimensional (3D) cultivation}

Different jejunum fragments from the small intestine were obtained from Hanwoo cows and collected in washing buffer containing ice-cold phosphate-buffered saline (PBS) containing

1\% penicillin/streptomycin (Sigma-Aldrich, NY, USA). The fragments were opened longitudinally and washed thoroughly with washing buffer to remove the debris. Mucosal and submucosal layers were gently scraped off using a glass slide. The remaining muscle layer was collected into a $50-\mathrm{ml}$ tube containing $30 \mathrm{ml}$ of washing buffer after being cut into 3- to 5-mm pieces, repeatedly washed by shaking vigorously and centrifuged at $300 \mathrm{~g}$ until the supernatant was clear. The collected pellet was resuspended in $25 \mathrm{ml}$ of Cell Disassociation Solution (StemCell Technologies, Vancouver, Canada) and incubated at room temperature for 40 min on a rocker to release the crypts. Crypts were collected after pipetting and 
centrifugation at $300 \mathrm{~g}$ for $5 \mathrm{~min}$. The pellet was resuspended in $1 \mathrm{ml}$ of intestinal human organoid medium (StemCell Technologies), and the crypts were counted under an inverted microscope. The seeding mix composed of medium with 100-150 crypts, and Matrigel in a 1:1 ratio was prepared and then placed in the middle of a 24-well plate. The cells were returned to an incubator to polymerize the Matrigel, and after $20 \mathrm{~min}, 1 \mathrm{ml}$ of organoid growth medium was gently added to each well.

\section{Passage and cryopreservation of bovine intestinal organoids}

Bovine intestinal organoids were subjected to passage approximately once a week upon maturation. Briefly, the medium was gently aspirated and rinsed with ice-cold PBS without disturbing the organoid dome. To harvest the organoids, a 10x volume of enzyme-free cell disassociation buffer $(1 \mathrm{ml})$ was added to a Matrigel dome $(100 \mu \mathrm{l})$ in each well and incubated for $10 \mathrm{~min}$ in an incubator. Organoids were dislodged by gentle pipetting and collected by centrifugation at $300 \mathrm{~g}$ for $5 \mathrm{~min}$. The pellet was resuspended in the desired amount of medium and Matrigel in a 1:1 ratio, and each well (140-150 organoids) was distributed into three parts in subsequent passages and seeded in 24-well plates. For cryopreservation, organoids were resuspended in preserving solution composed of $90 \%$ medium and $10 \%$ dimethyl sulfoxide (DMSO) (Sigma-Aldrich), stored at $-80^{\circ} \mathrm{C}$ for $24 \mathrm{hr}$ and transferred to a liquid nitrogen tank for long-term storage.

\section{Histology and immunohistochemistry}

The small intestine segments were fixed in 10\% neutral-buffered formalin (Sigma-Aldrich) after strong washing with ice-cold PBS. The segments were subsequently embedded in a paraffin block, and the paraffin-embedded intestinal tissue was vertically and horizontally sectioned at a thickness of 3-5 $\mu \mathrm{m}$. The sections were then deparaffinized in xylene, rehydrated with water via a graded alcohol series, and processed prior to haematoxylin and eosin (Merck, Darmstadt, Germany) staining. In the immunohistochemical analysis, the sections were permeabilized with $0.1 \%$ Triton X-100 in PBS for 5 min and incubated with $0.1 \%$ normal goat serum for $1 \mathrm{~h}$ to block nonspecific binding after antigen retrieval by boiling the sections in a sodium citrate buffer solution. The samples were incubated with appropriate dilutions of primary antibodies at $4^{\circ} \mathrm{C}$ overnight. The antibodies used in this study are shown in Table 1. After washing, the samples were reacted with anti-mouse and anti-rabbit secondary antibodies coupled to Alexa Fluor-488 and Alexa Fluor-594 (Molecular Probes/Life Technologies, Waltham, MA, USA), respectively, for $1 \mathrm{~h}$ at room temperature. These fluorescent samples were counterstained with diamidino2-phenylindole (DAPI). The images were captured using an Olympus X100 confocal microscope (Olympus, Tokyo, Japan). 
The organoids were maintained in 24-well plates until maturation. The fixation media was aspirated in the wells, and the organoids were washed thoroughly with cold PBS and incubated in neutrally buffered $4 \%$ paraformaldehyde (Sigma-Aldrich) for $30 \mathrm{~min}$ at room temperature. Then, the organoids were permeabilized in buffer containing $0.5 \%$ Triton X-100 (Sigma-Aldrich) in PBS for $30 \mathrm{~min}$ at room temperature. The blocking step was performed using $3 \%$ bovine serum albumin (BSA) in PBS for $1 \mathrm{~h}$ at room temperature. The organoids were thoroughly rinsed with PBS and incubated overnight at $4{ }^{\circ} \mathrm{C}$ with the appropriate primary antibodies, as shown in Table 1, at their appropriate dilutions. The marker gene expression was detected by incubating the samples with corresponding secondary antibodies coupled to AlexaFluor-488 and AlexaFluor-594 (Molecular Probes/Life Technologies) for $1 \mathrm{~h}$ at room temperature. These fluorescent samples were counterstained with diamidino-2-phenylindole (DAPI) and mounted on glass slides using ProLong Gold antifade (Life Technologies, Waltham, MA, USA) mounting medium. The images were captured under an Olympus X100 confocal microscope (Olympus).

\section{Epithelial barrier permeability assay using FITC-dextran}

Epithelial barrier function was tested by diluting powdered Fluorescein isothiocyanate (FITC)-dextran (4 and $40 \mathrm{kDa}$ ) (Sigma-Aldrich) in nuclease-free water, which resulted in a $1 \mathrm{mg} / \mathrm{ml}$ working solution. The organoids were placed in 24-well plates and allowed to grow until fully developed into crypt and villi structures. Then, $25 \mathrm{ng} / \mathrm{ml}$ FITC-dextran was added to each well, and the plate was incubated under normal growth conditions. The permeability was observed using luminal absorption and recorded for more than $180 \mathrm{~min}$ at 30-min intervals under a Leica CTR6000 fluorescence microscope (Leica, Wentzler, Germany).

\section{RNA isolation}

Total RNA for prepared samples including intestinal organoids was isolated using TRIzol reagent (Life Technologies, Carlsbad, CA, USA) as described previously $[14,15]$. RNA quality was assessed by an Agilent 2100 bioanalyzer using an RNA 6000 Nano Chip (Agilent Technologies, Amstelveen, The Netherlands), and RNA quantification was performed using an ND 2000 Spectrophotometer (Thermo Inc., DE, USA).

\section{Library preparation and sequencing}

For control and test RNAs, library construction was performed using QuantSeq 3' mRNA-Seq. Library Prep Kit (Lexogen, Inc., Austria) according to the manufacturer's instructions. In brief, each 500 ng of total RNA was prepared, an oligo-dT primer containing an Illumina-compatible sequence at its $5^{\prime}$ end was hybridized to the RNA, and reverse transcription was performed. After degradation of the RNA template, second 
strand synthesis was initiated by a random primer containing an Illumina-compatible linker sequence at its 5 ' end. The double-stranded library was purified using magnetic beads to remove all reaction components. The library was amplified to add the complete adapter sequences required for cluster generation. The finished library is purified from PCR components. High-throughput sequencing was performed as single-end 75 sequencing on a NextSeq 500 (Illumina, Inc., USA).

\section{Data analysis}

QuantSeq 3`mRNA-Seq. reads were aligned using Bowtie2 [16]. Bowtie2 indices were generated from either the genome assembly sequence or the representative transcript sequences for alignment to the genome and transcriptome. The alignment file was used for assembling transcripts, estimating their abundances and detecting differential expression of genes. Differentially expressed genes were determined based on counts from unique and multiple alignments using the coverage command in Bedtools [17]. The RC (read count) data were processed based on the quantile normalization method using EdgeR within R (R Development Core Team, 2016) using Bioconductor [18]. Gene classification was based on searches performed in the DAVID (http://david.abcc.ncifcrf.gov/) and Medline databases (http://www.ncbi.nlm.nih.gov/).

\section{Data availability}

QuantSeq 3' mRNA-Seq data sets are available via the following accession code in the Gene Expression Omnibus database (GEO): GSE163425

\section{Statistical analysis}

All data are expressed as the means \pm standard error of the mean (SEM) from three independent experiments. Significant differences between groups were analysed by one-way ANOVA or Student's ttest. A P value less than and equal to 0.05 indicated statistical significance ( ${ }^{*} P$ value $\left.\leq 0.05\right)$.

\section{Results}

\section{Long-term cultivation of bovine intestinal organoids}

Intestinal crypts were isolated from the small intestine (Jejunum) of healthy Hanwoo cattle (> 24 months old), sequentially embedded in Matrigel and cultivated in Intesticult medium. Figure 1A illustrates the experimental procedures for the isolation of intestinal crypts and the cultivation of bovine intestinal organoids. The recapitulating capacity of the organoids was demonstrated by the stable growth for more than passaged 10 (P10) and the long-term maintenance. As shown in Figure 1B, these organoids showed 
distinct crypt and villus structures (branched structures) surrounding the lumen from P1 to P4 generations at early passages. Subsequent detailed structures represent organoid propagation from day 0 to the fully-grown structure on day 8 after isolation and cultivation of intestinal crypts from small intestine (Supplementary Figure S1). Furthermore, they showed healthy and consistent growth in an average of 130-150 organoids per basement matrix dome from P1 to P10 at each generation, indicating the recapitulating capacity of the crypt (Figure 1C). At this density, bovine intestinal organoids showed healthy and consistent growth. Furthermore, intestinal organoids at P5 were identified as positive against Ki67, a proliferating cell marker (Figure 1D). Collectively, these results demonstrated that bovine intestinal organoids cultivated and isolated from small intestine (Jejunum) crypts were maintained long-term without loss of the recapitulating capacity of crypts.

\section{Identification of intestinal stem cells from bovine small intestine}

To search for position effects for efficient isolation and cultivation of intestinal organoids from the small intestine, we selected four different locations in the jejunum between the duodenum and ileum. Initially, intestinal tissue sections were subjected to anatomical analysis using haematoxylin and eosin histological staining to identify distinct crypt and villus structures. As shown in Figure $2 \mathrm{~A}$, locations \#1 and \#2 were better developed than locations \#3 and \#4. The detailed view from vertical and horizontal sections in locations \#1 and \#2 showed integral structures of the intestinal epithelium gland, such as crypts at the bottom and finger-shaped villi on the apical side (Supplementary Figure S2). Furthermore, to verify the efficiency of the derivation of intestinal organoids from four different locations, we subsequently cultivated intestinal organoids. Based on the results, the number of intestinal organoids per basement matrix dome was highest in location \#1 (Figure 2B), indicating the most growth potential for derivation of intestinal organoids. In addition, to identify intestinal stem cells in vivo, immunohistochemistry with respect to several markers involved in intestinal stem cells and epithelial cells was conducted. As shown in Figure $2 \mathrm{C}$, intestinal crypts isolated from location \#1 of the small intestine had distinct expression, such as leucine-rich repeat containing $\mathrm{G}$ protein-coupled receptor 5 (LGR5), a key gene required for stemness that is expressed in columnar crypt cells, B lymphoma Mo-MLV insertion region 1 homology (Bmi1), which was found in +4 cells adjacent to paneth cells and F-actin in the intestinal epithelial cytoskeleton. Moreover, the fluorescently stained crypts showed epitheliumspecific expression of Mucin2 in goblet cells, E-cadherin in adherent junctions (Supplementary Figure S3). Together, these results are the first to show the identification of LGR5 ${ }^{+}$intestinal stem cells from the small intestine and demonstrate the regional differences for efficient generation of intestinal organoids in bovine.

\section{Characterization and paracellular permeability of bovine intestinal organoids}


To characterize the cellular potential of bovine intestinal organoids derived from the small intestines of adults, we investigated the spatial expression of several specific markers involved in intestinal stem cells and epithelium characteristics in bovine intestinal organoids at passaged 5 . As shown in Figure 3A, the organoids had distinct expression, such as LGR5 and Bmi1. Moreover, the fluorescently stained organoids showed epithelium-specific expression against Mucin2 for goblet cells that contributes to epithelial barrier integrity, E-cadherin for adherent junctions, F-actin for intestinal epithelial cytoskeleton, Chromogranin A for enteroendocrine cells and Cytokeratin 19 for enterocytes, indicating that the concomitant expression of intestinal epithelial genes in intestinal organoids derived from intestinal crypts mimicked the topology of an in vivo intact intestine. Furthermore, intestinal organoids at P5 were immunoreactive to antibodies against several representative nutrient absorption markers, especially sodium-dependent glucose transporter (SGLT1), proton-coupled peptide transporter (PEPT1), glucose transporter (Glut2), glucagon-like peptide 1 (GLP1) and bile acid receptor (TGR5) (Figure 3B). In addition, we investigated the paracellular permeability character of the epithelial layer using fluorescent tracers up to $4 \mathrm{hr}$ after treatment. FITC-dextran labelled the organoid lumen, demonstrating a high permeability for compounds of up to $4 \mathrm{kDa}$, such as glucose, peptides and fatty acids, while FITC-dextran $40 \mathrm{kDa}$ failed to enter the organoid lumen (Figure $3 \mathrm{C}$ ). Concentration of FITC was maintained constantly and slow diffusion of FITC-dextran $4 \mathrm{kDa}$ start to enter the organoid lumen at the $1.5 \mathrm{hr}$ (Supplementary Figure S4), indicating the presence of a mucous layer, which plays a major role in barrier function and nutrient absorption. The mucosal barrier function results showed that there was mucins secretion by goblet cells. Together, these functional testing results suggested that intestinal organoids were physiological relevance to the in vivo gut absorption properties.

\section{Large-scale gene expression profiling of bovine intestinal organoids}

To investigate the genetic properties of bovine intestinal organoids for large-scale gene expression profiling, QuantSeq 3' mRNA-Seq. Library was constructed. As shown in Figure 4A, principal component analysis (PCA) indicated that the distance between intestinal organoids and the small intestine was relatively close compared to muscle in bovine. In addition, the heatmap showed that many genes in epithelium-characteristic categories, such as tight junctions, adherent junctions, desmosomes and gap junctions, were significantly expressed in intestinal organoids at P5 and P10 and in the small intestine compared to muscle as a control (Figure 4B). Furthermore, scatter plot revealed that many genes related to intestinal stem cell markers such as $L G R 5$, Achaete-Scute Family BHLH Transcription Factor 2 (ASCL2), EPH Receptor B2 (EPHB2), Pleckstrin Homology like Domain Family A Member 1 (PHLDA1), SRY-Box Transcription Factor 9 (SOX9) and Olfactomedin 4 (OLFM4) were significantly upregulated in intestinal organoids at P5 or similar between intestinal organoids and the intestine (Figure 4C). Taken together, these results indicated that the genetic properties of bovine intestinal organoids were highly similar to those of in vivo.

\section{Discussion}


Recently, intestinal organoids have evolved as potential alternatives to in vivo systems [6, 19], and has been a focus of research in livestock species including bovine, swine and chicken $[4,5,10,20]$. Especially, these organoids has been shown to be an attractive model for mucosal permeability, enabling us to investigate the interactions of pathogenic bacteria, viruses, nutrient absorption and the maintenance of host homeostasis with the gut epithelium of their host [21-24].

In this study, we successfully established intestinal organoids derived intestinal crypts from small intestine and reported a reliable method for robust 3D expansion of intestinal stem cells in bovine. Firstly, we isolated intestinal crypts that included intestinal stem cells from the small intestine (jejunum) in adult bovines and cultivated them using on the scaffold-based method. The organoids representing distinct crypt and villus structures surrounding the lumen can be long-term maintained (> P10) during several passages without loss of their recapitulating capacity. Furthermore, we achieved a consistent growth rate of 130-150 per basement matrix dome and identified as positive against Ki67, a proliferating cell marker [25] in intestinal organoids at P5 (Figure 1). The results of the current study suggest that the organoids closely mimicked the in vivo organ physiology and remained indefinitely intact under controlled conditions without loss of the recapitulating capacity of crypts.

Next, to assess the position effects for efficient isolation and derivation of intestinal organoids along the length of the small intestine in bovine, we sectioned four different locations in the jejunum between the duodenum and ileum. Interestingly, we found that jejunum (location \#1 and \#2) close to the duodenum showed integral structures of the intestinal epithelium gland, such as crypts at the bottom and fingershaped villi on the apical side and intestinal crypts isolated from location \#1 in the jejunum close to the duodenum had the most growth potential for derivation of intestinal organoids (Figure 2). However, mouse models have shown that more distal tissues in fetal intestine can be formed organoids well [26], suggesting the regional differences in vitro growth potential. Therefore, it seems likely that such information would be valuable for derivation intestinal organoids and robust 3D expansion due to differences between species or ages. In addition, in search for identification of intestinal stem cells from small intestine in bovine in details, we confirmed that the intestinal crypts isolated from the small intestine in bovine had distinct expression, such as leucine-rich repeat containing $\mathrm{G}$ protein-coupled receptor 5 (LGR5), a key gene required for stemness that is expressed in columnar crypt cells, $B$ lymphoma Mo-MLV insertion region 1 homology (Bmi1), which was found in +4 cells adjacent to paneth cells and F-actin in the intestinal epithelial cytoskeleton, epithelium-specific expression of Mucin2 in goblet cells, E-cadherin in adherent junctions, supporting previous studies on mouse and human intestinal organoids such as the mini-gut $[8,25]$. Collectively, our findings demonstrate for the first time that the identification of LGR5 ${ }^{+}$intestinal stem cells from the small intestine and demonstrate the regional differences for efficient derivation of intestinal organoids in bovine.

With regard to the cellular potential of bovine intestinal organoids, we investigated the several specific markers involved in intestinal stem cells and epithelium's characteristics. As shown in Figure 3, bovine intestinal organoids had distinct expression, such as LGR5 and Bmi1 for self-renewal capacities, and also showed epithelium-specific expression against Mucin2, indicating the presence of mucin secreting in 
goblet cells that lined up the epithelial mucosa, E-cadherin for adherent junctions, F-actin for intestinal epithelial cytoskeleton, Chromogranin A for enteroendocrine cells and Cytokeratin 19 for enterocytes representing the cellular diversity composed of the intestinal epithelium. It is well accepted that intestinal organoids consists of serveral intestinal cellular types including intestinal stem cells, paneth cells, enteroendocrine cells, goblet cells, transit amplifying cells, and enterocytes [2]. Based on our results, the cellular potential of intestinal organoids derived from the small intestines of adult bovine has quite resemblance to in vivo small intestine and is similar to that of human intestinal organoids [27]. In addition, the intestinal epithelium plays important roles in nutrient absorption across the membrane and the diffusion of small molecules across the intestinal barrier, thus ensuring health by nutrient absorption and preventing bacterial translocation via the bloodstream $[28,29]$. Therefore, we further investigated the several representative nutrient absorption markers and paracellular permeability character of the epithelial layer [30]. As shown in Figure 3B and 3C, we found that intestinal organoids at P5 had the specific-expression of sodium-dependent glucose transporter (SGLT1), proton-coupled peptide transporter (PEPT1), glucose transporter (Glut2), glucagon-like peptide 1 (GLP1) and bile acid receptor (TGR5), and showed a high permeability for compounds of up to $4 \mathrm{kDa}$, such as glucose, peptides and fatty acids, while FITC-dextran $40 \mathrm{kDa}$ failed to enter the organoid lumen, indicating the absorptive capacity of bovine intestinal organoids. Taken together, these results indicate that bovine intestinal organoids preserve the functional and phenotypic characteristics of the small intestine and can be used as physiological indicators of nutrient absorption in nutritional research showing mimicked the topology of an in vivo intact intestine.

With regard to the genetic properties of bovine intestinal organoids using large-scale gene expression profiling, we found that the distance between intestinal organoids and the small intestine was relatively close compared to muscle in bovine and many genes in epithelium-characteristic categories [24], such as tight junctions, adherent junctions, desmosomes and gap junctions, were significantly expressed in intestinal organoids at P5 and P10 (Figures 4), suggesting the physiological similarity between the intestinal organoids and small intestine. In addition, they had significant expression many genes related to intestinal stem cells such LGR5, Achaete-Scute Family BHLH Transcription Factor 2 (ASCL2), EPH Receptor B2 (EPHB2), Pleckstrin Homology like Domain Family A Member 1 (PHLDA1), SRY-Box Transcription Factor 9 (SOX9) and Olfactomedin 4 (OLFM4). Collectively, our findings suggested that the genetic properties of bovine intestinal organoids were highly similar to those of in vivo.

\section{Conclusion}

These results provide a reliable method for efficient isolation of intestinal crypts from small intestine and robust 3D expansion of intestinal stem cells in adult bovine and demonstrate the in vitro 3D organoids mimics the in vivo tissue topology and functionality. Finally, intestinal organoids are potential alternatives to in vivo system and will facilitate the practical use of a model to replace animal experiments in the fields of animal biotechnology for various purposes. 


\section{Abbreviations}

3D, Three-dimensional; 2D, Two-dimensional; ASCs, Adult stem cells; PBS, phosphate-buffered saline; DMSO, dimethyl sulfoxide; DAPI, diamidino-2-phenylindole ; BSA, bovine serum albumin; FITC, Fluorescein isothiocyanate; SEM, standard error of the mean; P, passaged; LGR5, leucine-rich repeat containing G protein-coupled receptor 5; Bmi1, B lymphoma Mo-MLV insertion region 1 homology; SGLT1, sodium-dependent glucose transporter; PEPT1, proton-coupled peptide transporter; Glut2, glucose transporter; GLP1, glucagon-like peptide 1; TGR5, bile acid receptor; PCA, principal component analysis; ASCL2, Achaete-Scute Family BHLH Transcription Factor 2; EPHB2, EPH Receptor B2; PHLDA1, Pleckstrin Homology like Domain Family A Member 1; SOX9, SRY-Box Transcription Factor 9; OLFM4, Olfactomedin 4

\section{Declarations}

\section{Ethics approval and consent to participate}

Experimental use of Hanwoo cattle were approved by the Institutional Animal Care and Use Committee (IACUC) of the National Institute of Animal Science.

\section{Consent for publication}

Not applicable.

\section{Availability of data and material}

The datasets during and/or analyzed during the current study available from the corresponding authors on reasonable request.

\section{Competing interests}

The authors declare that they have no competing interests.

\section{Funding}

This work was supported with the National Institute of Animal Science (Grant No. PJ01422201), Rural Development Administration (RDA), Korea. 


\section{Authors' contributions}

LBR participated in study design and coordination. LBR and IH participated in the design of the study, carried out the experiments, statistical analysis and wrote manuscript. YH, LSI, WH, LHC, OSA and LP were involved in data interpretation. RJG participated in writing the final versions of the manuscript. All authors have read and approved the final manuscript.

\section{Acknowledgements}

We would like to sincerely thank Animal Products Research and Development Division, National Institute of Animal Science for helping sample collection in Hanwoo cattle.

\section{Open access}

This article is distributed under the terms of the Creative Commons Attribution 4.0 International License (http://creativecommons.org/licenses/by/4.0/), which permits unrestricted use, distribution, and reproduction in any medium, provided you give appropriate credit to the original author(s) and the source, provide a link to the Creative Commons license, and indicate if changes were made. The Creative Commons Public Domain Dedication waiver (http://creativecommons.org/publicdomain/zero/1.0/) applies to the data made available in this article, unless otherwise stated.

\section{References}

1. Kretzschmar K, Clevers H: Organoids: Modeling Development and the Stem Cell Niche in a Dish. Dev Cell 2016, 38(6):590-600.

2. Rahmani S, Breyner NM, Su HM, Verdu EF, Didar TF: Intestinal organoids: A new paradigm for engineering intestinal epithelium in vitro. Biomaterials 2019, 194:195-214.

3. Wallach TE, Bayrer JR: Intestinal Organoids: New Frontiers in the Study of Intestinal Disease and Physiology. J Pediatr Gastroenterol Nutr 2017, 64(2):180-185.

4. Hamilton CA, Young R, Jayaraman S, Sehgal A, Paxton E, Thomson S, Katzer F, Hope J, Innes E, Morrison LJ et al: Development of in vitro enteroids derived from bovine small intestinal crypts. Vet Res 2018, 49(1):54.

5. Li Y, Yang N, Chen J, Huang X, Zhang N, Yang S, Liu G, Liu G: Next-Generation Porcine Intestinal Organoids: an Apical-Out Organoid Model for Swine Enteric Virus Infection and Immune Response Investigations. J Viro/ 2020, 94(21). 
6. Marrella A, Buratti P, Markus J, Firpo G, Pesenti M, Landry T, Ayehunie S, Scaglione S, Kandarova H, Aiello M: In vitro demonstration of intestinal absorption mechanisms of different sugars using 3D organotypic tissues in a fluidic device. ALTEX 2020, 37(2):255-264.

7. Lancaster MA, Knoblich JA: Generation of cerebral organoids from human pluripotent stem cells. Nat Protoc 2014, 9(10):2329-2340.

8. Sato T, Vries RG, Snippert HJ, van de Wetering M, Barker N, Stange DE, van Es JH, Abo A, Kujala P, Peters PJ et al: Single Lgr5 stem cells build crypt-villus structures in vitro without a mesenchymal niche. Nature 2009, 459(7244):262-265.

9. Rallabandi HR, Yang H, Oh KB, Lee HC, Byun SJ, Lee BR: Evaluation of Intestinal Epithelial Barrier Function in Inflammatory Bowel Diseases Using Murine Intestinal Organoids. Tissue Eng Regen Med 2020, 17(5):641-650.

10. Derricott H, Luu L, Fong WY, Hartley CS, Johnston LJ, Armstrong SD, Randle N, Duckworth CA, Campbell BJ, Wastling JM et al: Developing a 3D intestinal epithelium model for livestock species. Cell Tissue Res 2019, 375(2):409-424.

11. Baek Y-C, Kim M, Jeong J-Y, Oh Y-K, Lee S-D, Lee Y-K, Ji S-Y, Choi H: Effects of Short-term Acute Heat Stress on Physiological Responses and Heat Shock Proteins of Hanwoo Steer (Korean Cattle). J Anim Reprod Biotechnol 2019, 34(3):173-182.

12. Miyazawa K, Hondo T, Kanaya T, Tanaka S, Takakura I, Itani W, Rose MT, Kitazawa H, Yamaguchi T, Aso $\mathrm{H}$ : Characterization of newly established bovine intestinal epithelial cell line. Histochem Cell Biol 2010, 133(1):125-134.

13. Zhan K, Lin M, Liu MM, Sui YN, Zhao GQ: Establishment of primary bovine intestinal epithelial cell culture and clone method. In Vitro Cell Dev Biol Anim 2017, 53(1):54-57.

14. Lee $B R$, Rengaraj $D$, Choi $H J$, Han JY: A novel F-box domain containing cyclin $F$ like gene is required for maintaining the genome stability and survival of chicken primordial germ cells. FASEB J 2020, 34(1):1001-1017.

15. Lee BR, Kim H, Park TS, Moon S, Cho S, Park T, Lim JM, Han JY: A set of stage-specific gene transcripts identified in EK stage $X$ and $\mathrm{HH}$ stage 3 chick embryos. BMC Dev Biol 2007, 7:60.

16. Langmead B, Salzberg SL: Fast gapped-read alignment with Bowtie 2. Nat Methods 2012, 9(4):357359.

17. Quinlan AR, Hall IM: BEDTools: a flexible suite of utilities for comparing genomic features. Bioinformatics 2010, 26(6):841-842.

18. Gentleman RC, Carey VJ, Bates DM, Bolstad B, Dettling M, Dudoit S, Ellis B, Gautier L, Ge Y, Gentry J et al: Bioconductor: open software development for computational biology and bioinformatics. Genome Biol 2004, 5(10):R80.

19. Belair DG, Visconti RJ, Hong M, Marella M, Peters MF, Scott CW, Kolaja KL: Human ileal organoid model recapitulates clinical incidence of diarrhea associated with small molecule drugs. Toxicol In Vitro 2020, 68:104928. 
20. Pierzchalska M, Grabacka M, Michalik M, Zyla K, Pierzchalski P: Prostaglandin E2 supports growth of chicken embryo intestinal organoids in Matrigel matrix. Biotechniques 2012, 52(5):307-315.

21. Forbester JL, Goulding D, Vallier L, Hannan N, Hale C, Pickard D, Mukhopadhyay S, Dougan G: Interaction of Salmonella enterica Serovar Typhimurium with Intestinal Organoids Derived from Human Induced Pluripotent Stem Cells. Infect Immun 2015, 83(7):2926-2934.

22. Saxena K, Blutt SE, Ettayebi K, Zeng XL, Broughman JR, Crawford SE, Karandikar UC, Sastri NP, Conner ME, Opekun AR et al: Human Intestinal Enteroids: a New Model To Study Human Rotavirus Infection, Host Restriction, and Pathophysiology. J Virol 2016, 90(1):43-56.

23. Peterson LW, Artis D: Intestinal epithelial cells: regulators of barrier function and immune homeostasis. Nat Rev Immunol 2014, 14(3):141-153.

24. Pearce SC, Al-Jawadi A, Kishida K, Yu S, Hu M, Fritzky LF, Edelblum KL, Gao N, Ferraris RP: Marked differences in tight junction composition and macromolecular permeability among different intestinal cell types. BMC Bio/ 2018, 16(1):19.

25. Sato T, Stange DE, Ferrante M, Vries RG, Van Es JH, Van den Brink S, Van Houdt WJ, Pronk A, Van Gorp J, Siersema PD et al: Long-term expansion of epithelial organoids from human colon, adenoma, adenocarcinoma, and Barrett's epithelium. Gastroenterology 2011, 141(5):1762-1772.

26. Fordham RP, Yui S, Hannan NR, Soendergaard C, Madgwick A, Schweiger PJ, Nielsen OH, Vallier L, Pedersen RA, Nakamura $T$ et al: Transplantation of expanded fetal intestinal progenitors contributes to colon regeneration after injury. Cell Stem Cell 2013, 13(6):734-744.

27. Fujii M, Matano M, Toshimitsu K, Takano A, Mikami Y, Nishikori S, Sugimoto S, Sato T: Human Intestinal Organoids Maintain Self-Renewal Capacity and Cellular Diversity in Niche-Inspired Culture Condition. Cell Stem Cell 2018, 23(6):787-793 e786.

28. Hill DR, Huang S, Tsai YH, Spence JR, Young VB: Real-time Measurement of Epithelial Barrier Permeability in Human Intestinal Organoids. J Vis Exp 2017(130).

29. Kong S, Zhang YH, Zhang W: Regulation of Intestinal Epithelial Cells Properties and Functions by Amino Acids. Biomed Res Int 2018, 2018:2819154.

30. Zietek T, Rath E, Haller D, Daniel H: Intestinal organoids for assessing nutrient transport, sensing and incretin secretion. Sci Rep 2015, 5:16831.

\section{Table}

Table 1. Antibodies used in this study for characterization of bovine intestinal organoids. 


\begin{tabular}{|lll|}
\hline Antibody & Host species & Company (Catalog No.) \\
\hline LGR5 & Mouse & Origene Technologies, Inc. (TA503316) \\
\hline Bmi1 & Rabbit & abcam (ab38295) \\
\hline Mucin2 & Mouse & Santa Cruz Biotechnology, Inc. (SC-515032) \\
\hline E-Cadherin & Mouse & BD Biosciences (61081) \\
\hline Cytokerain 19 & Rabbit & abcam (ab84632) \\
\hline F-actin & Rabbit & abcam (ab83746) \\
\hline Chromogranin A & Rabbit & abcam (ab85554) \\
\hline Glut2 & Rabbit & Novus Biologicals (NBP1-69466) \\
\hline PEPT1 & Rabbit & Bioss Antibodies (BS-0689R) \\
\hline SGLT1 & Rabbit & Novus Biologicals (NBP2-20338) \\
\hline GLP1 & Rabbit & MyBioSource (MBS2107860) \\
\hline TGR5 & Rabbit & abcam (ab72608) \\
\hline Ki67 & Rabbit & Cell Signaling Technology (D3B5) \\
\hline
\end{tabular}

\section{Figures}


A

\section{Bovine Intestinal Stem Cell Isolation}

1. Bovine small Intestine Fragment

2.

Dissect, Flush and Washing

3.

Gentle cell dissociation

4.

Crypt pipetting \& Filtration

5.

Matrigel Mix (1:1)

6.

Intestinal Organoid Medium

C

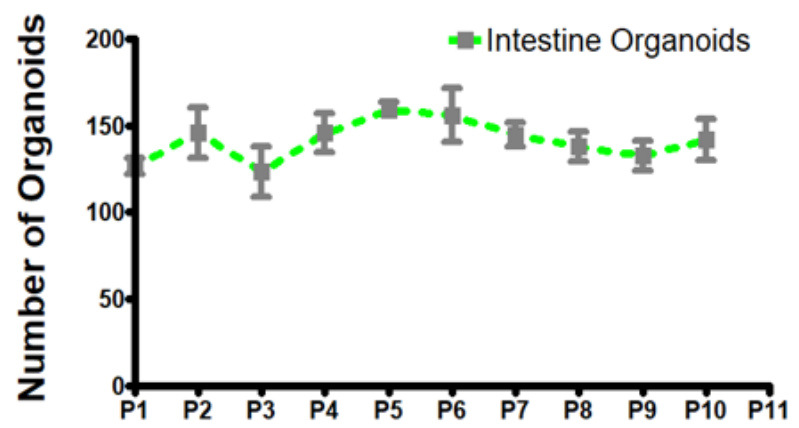

B

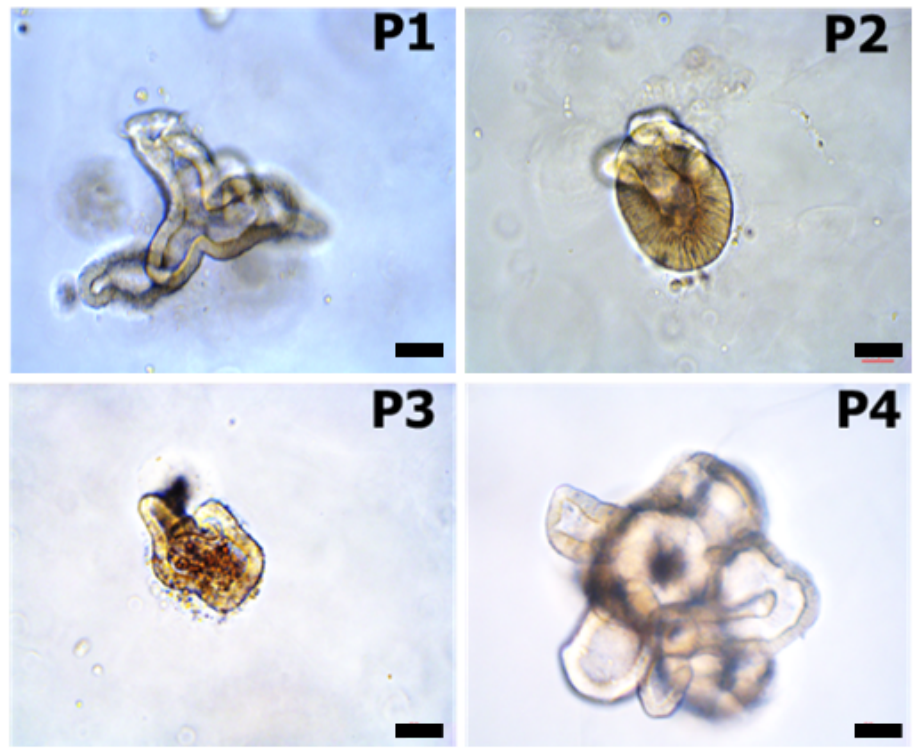

D
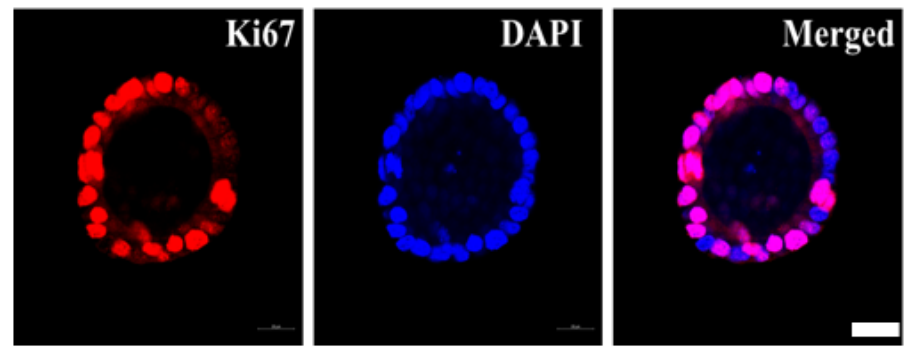

\section{Figure 1}

Isolation of intestinal crypts and three-dimensional (3D) cultivation of intestinal crypts including intestinal stem cells in bovine. (A) Experimental procedures regarding the isolation of intestinal crypts from the small intestine and the three-dimensional (3D) cultivation of intestinal crypts including intestinal stem cells in bovine. (B) Robust 3D expansion of intestinal crypts including bovine intestinal stem cells (P1-P4). The organoids can be spheroidal (round shaped), show budding (spheroids with extension) and have mature villi and crypt-like structures (branched structures). Scale bar: $50 \mu \mathrm{m}$. (C) Growth rate graph of bovine intestinal organoids showing the number of organoids/well (mean $n=3$ wells) growing in a 100 $\mu \mathrm{l}$ Matrigel dome in each well. Intestinal organoids were maintained for up to 10 generations without loss of the recapitulating capacity of crypts. (D) Intestinal organoids were immunostained for Ki67 at passaged 5 , a marker of proliferating cells and were counterstained with diamidino-2-phenylindole (DAPI). Scale bar: $20 \mu \mathrm{m}$. 
A

\#1
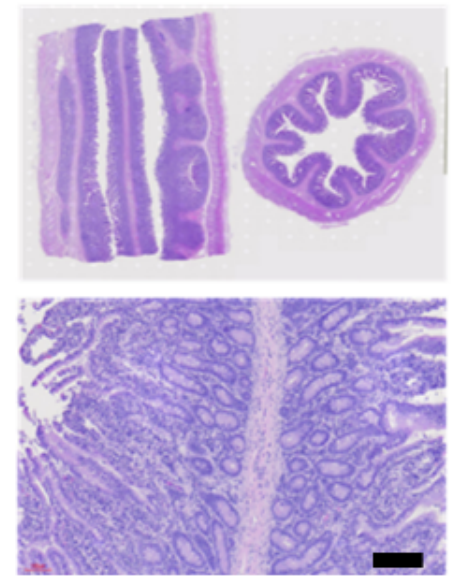

B

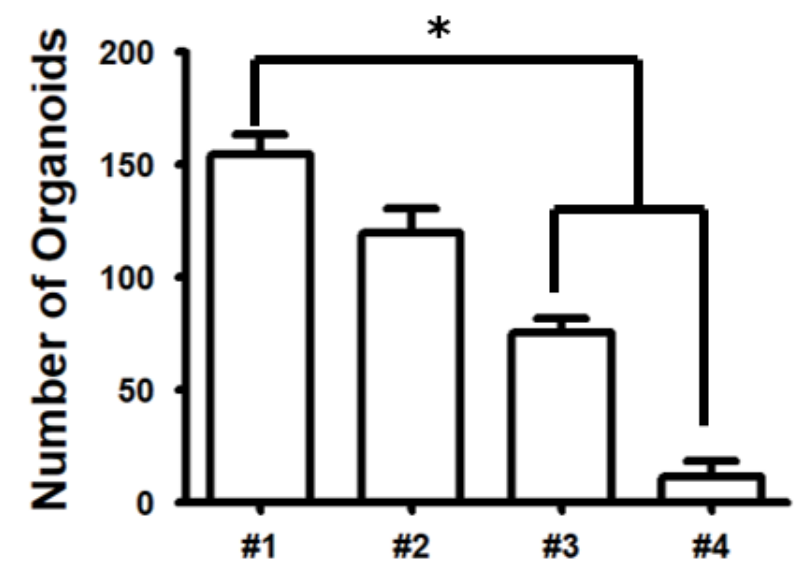

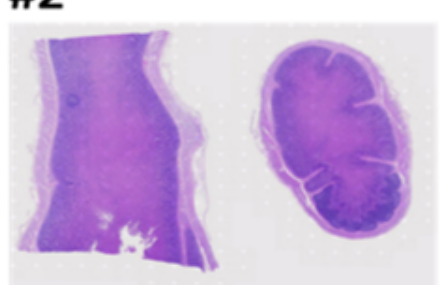

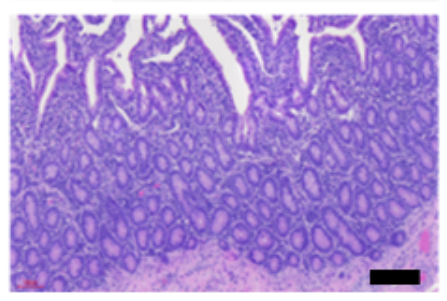

C
\#3

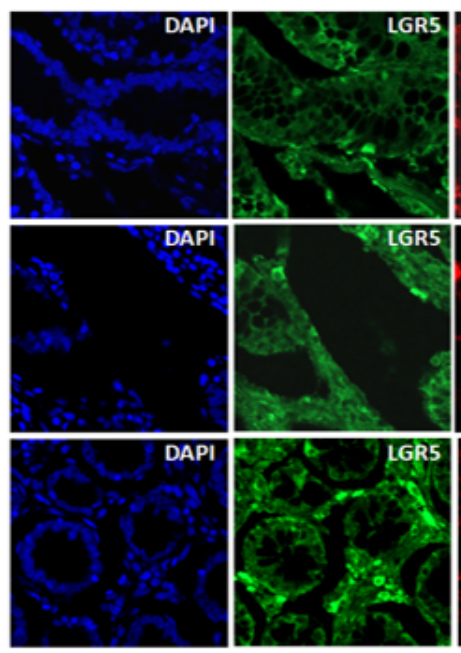

\#4
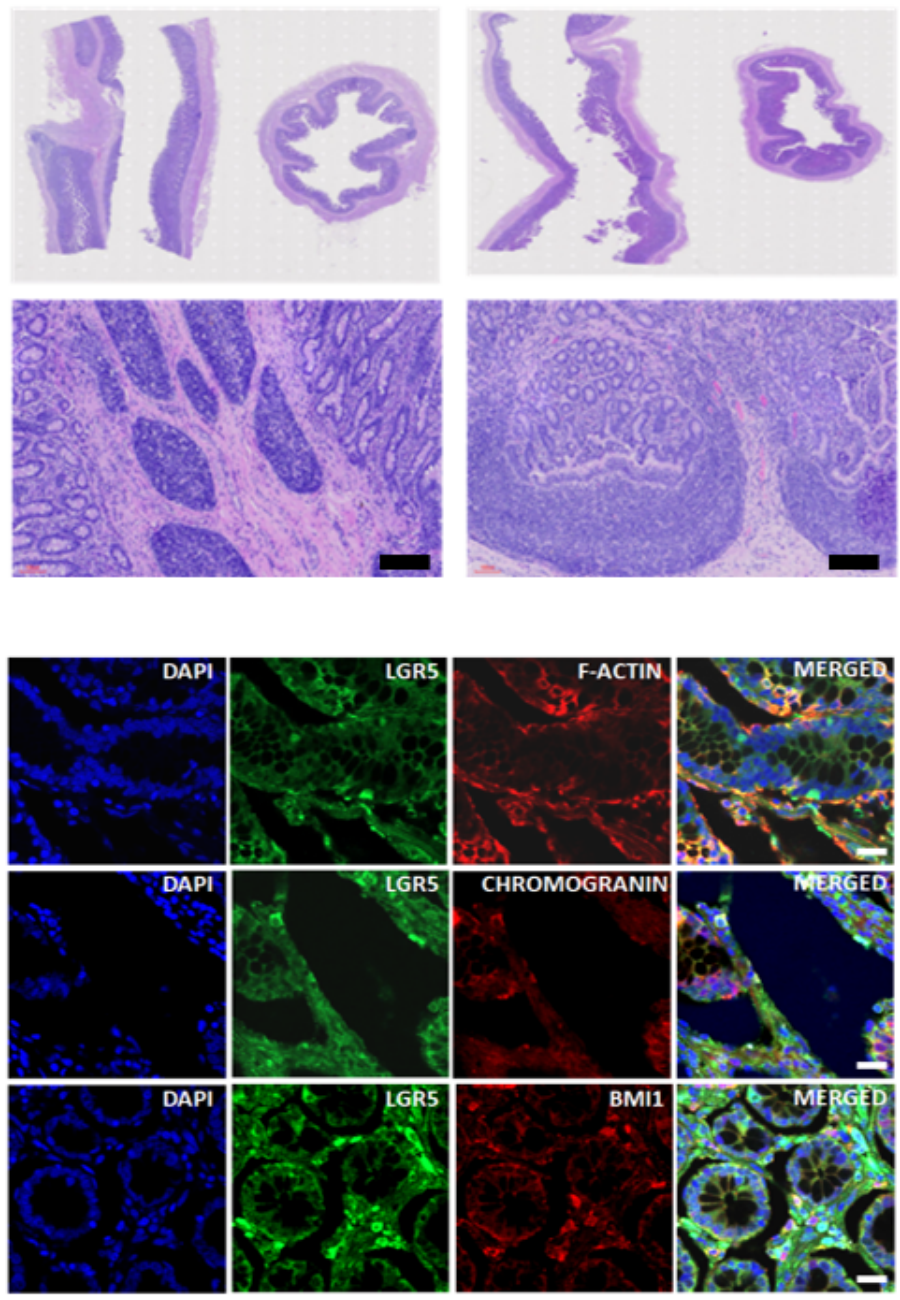

\section{Figure 2}

Immunohistochemical analysis of bovine small intestine. (A) Haematoxylin and eosin histological staining to identify distinct crypt and villus structures from four different locations (\#1, \#2, \#3 and \#4) in the jejunum between the duodenum and ileum. Scale bar: $300 \mu \mathrm{m}$. (B) The number of intestinal organoids per basement matrix dome to verify the efficiency of the derivation of intestinal organoids from four different locations. The number of intestinal organoids derived from location $\# 1$ in the jejunum close to the duodenum was significant higher compared to location \#3 and \#4. The values are the means plus the standard error of mean (S.E.M), and a p-value $<0.05\left(^{*}\right)$ was considered to be significant. (C) Immunohistochemistry of LGR5, Bmi1, F-actin and Chromogranin A in bovine small intestine. The fluorescently stained crypts were counterstained with diamidino-2-phenylindole (DAPI). Scale bar: $20 \mu \mathrm{m}$. 
A
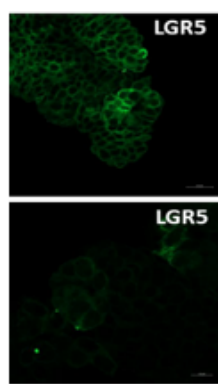

E-cadherin

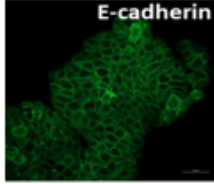

Mucin2
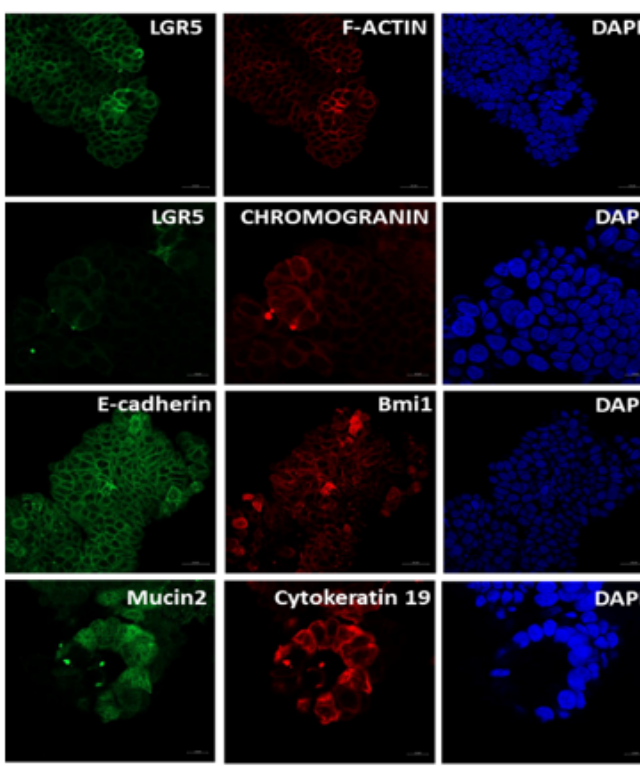

C

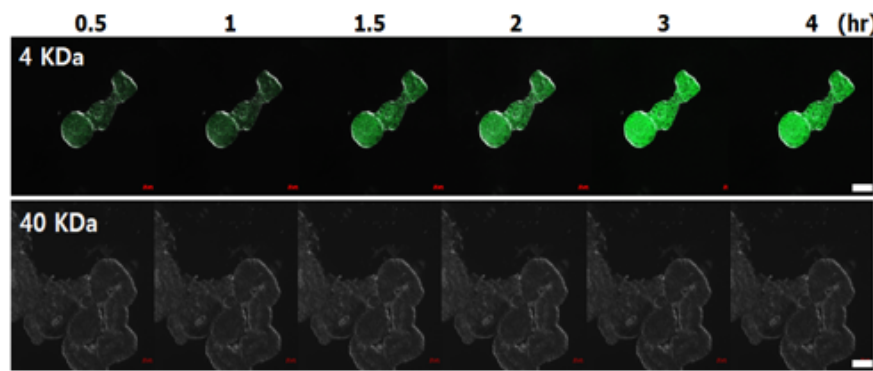

B
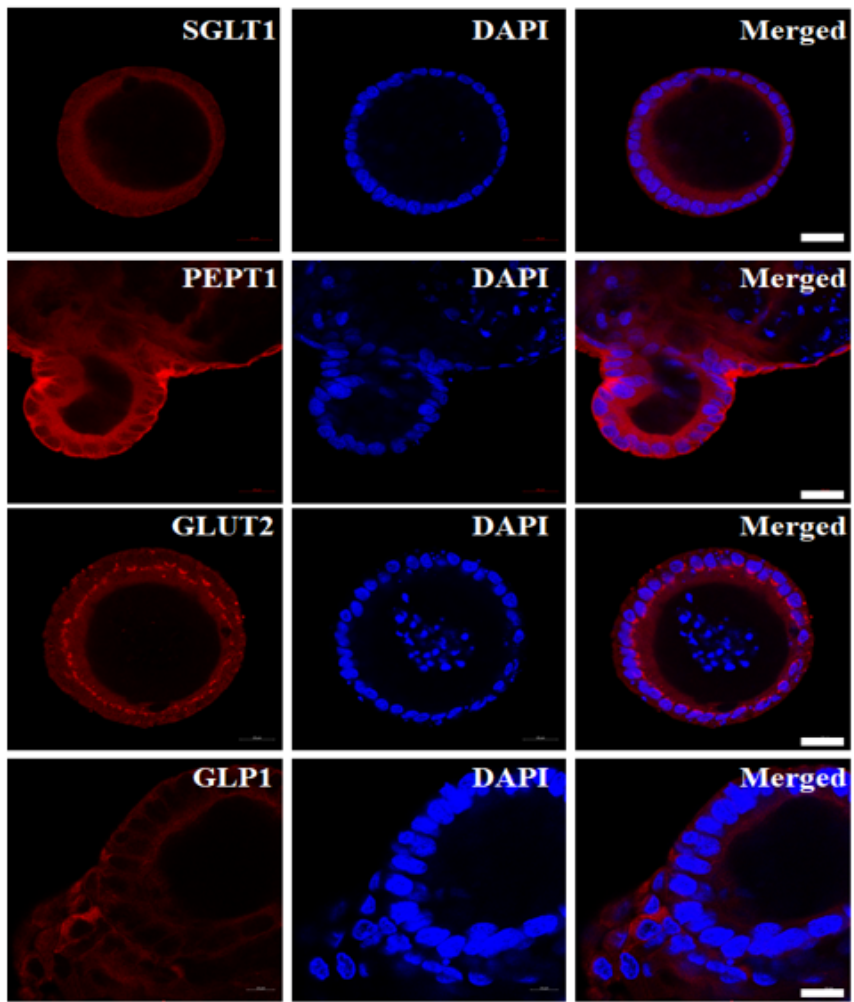

DAPI
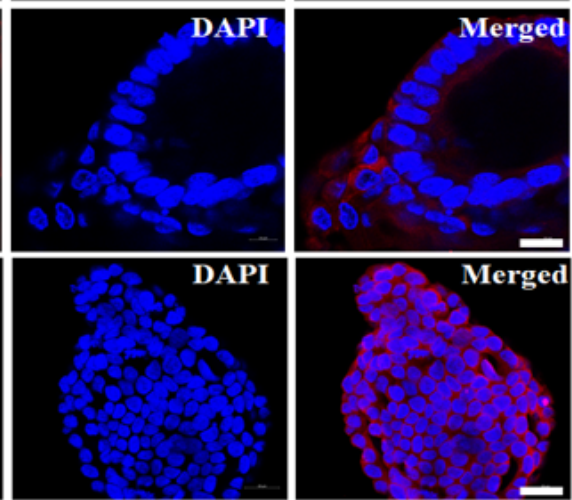

Figure 3

Characterization and paracellular permeability of bovine intestinal organoids. (A) Immunostaing of LGR5, Bmi1, F-actin, E-cadherin, Chromogranin A and Mucin2 in bovine intestinal organoids at passaged 5. The organoids were counterstained with DAPI. Scale bar: $20 \mu \mathrm{m}$. (B) Immunostaining of several representative nutrient absorption markers such as sodium-dependent glucose transporter (SGLT1), proton-coupled peptide transporter (PEPT1), glucose transporter (Glut2), glucagon-like peptide 1 (GLP1) and bile acid receptor (TGR5) in bovine intestinal organoids at passaged 5. The organoids were counterstained with DAPI. Scale bar: $20 \mu \mathrm{m}$. (C) Paracellular permeability of the epithelial layer in bovine intestinal organoids using fluorescent tracers. Intestinal organoids were treated with FITC-dextran to assess barrier function. FITC-dextran $4 \mathrm{kDa}$ showed high permeability, while FITC-dextran $40 \mathrm{kDa}$ failed to enter the organoid lumen. Scale bar: $20 \mu \mathrm{m}$. 
A

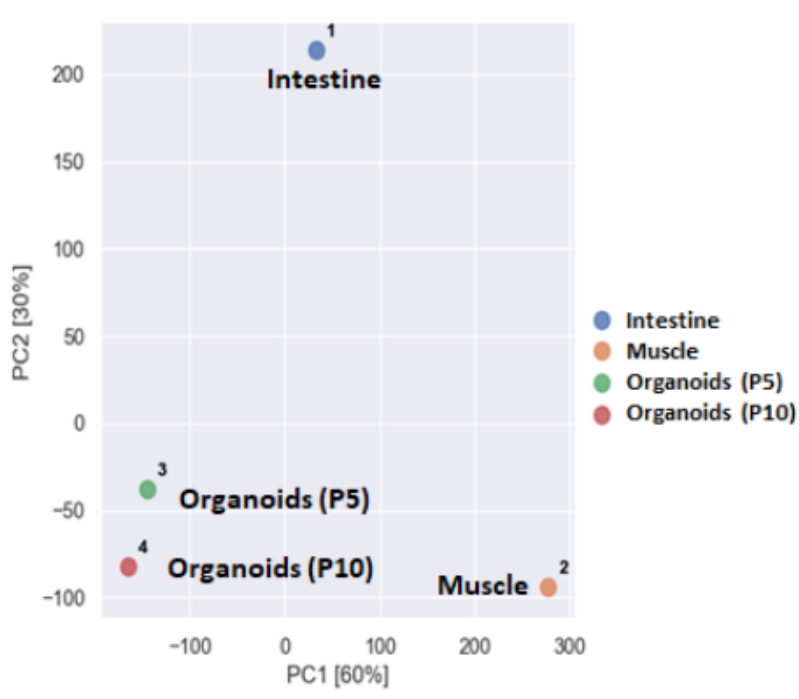

C

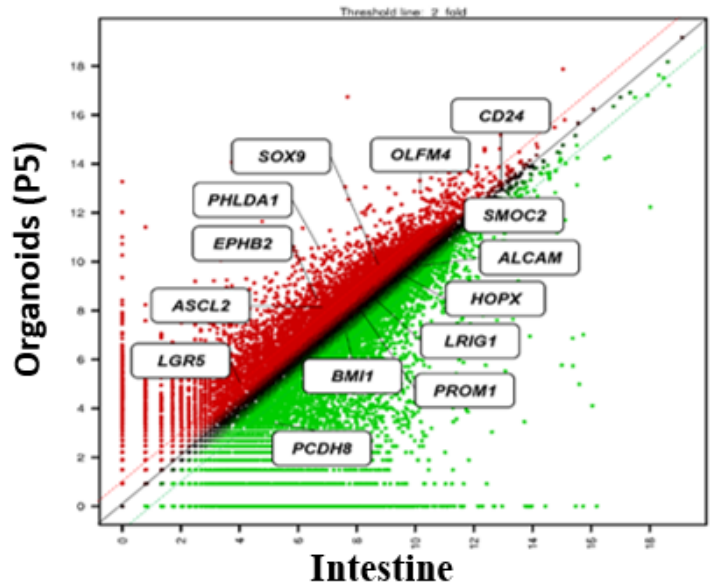

B

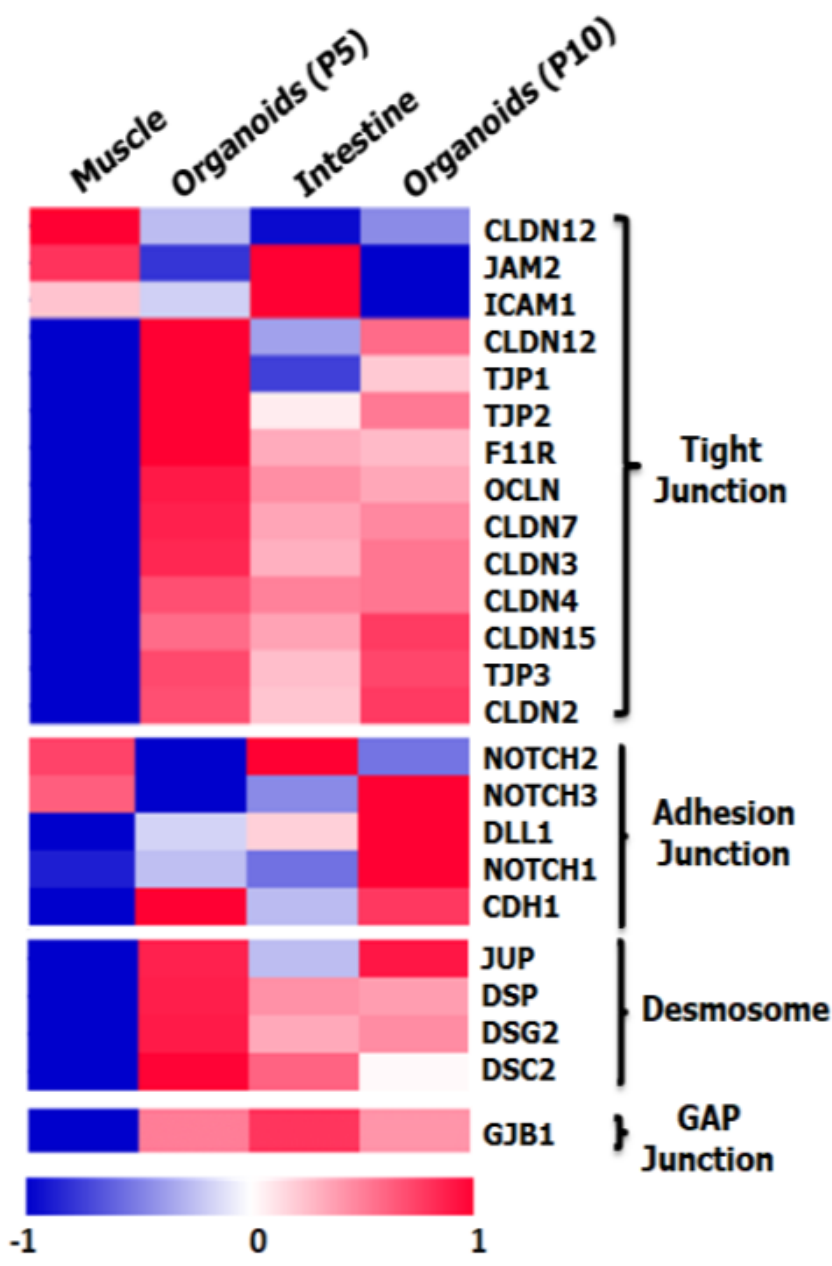

Figure 4

Gene expression profiling of bovine intestinal organoids using QuantSeq 3' mRNA-Seq. Library. (A) Principal component analysis (PCA) of intestinal organoids (P5), intestinal organoids (P10), small intestine and muscle in bovine. The distance between intestinal organoids and the small intestine was relatively close compared to muscle. (B) Heatmap showing many genes in epithelium-characteristic categories such as tight junctions, adherent junctions, desmosomes and gap junctions. Intestinal organoids at P5 and P10 and in the small intestine were significantly expressed compared to muscle as a control. (C) Scatter plot showing many genes related to intestinal stem cell markers such as LGR5, ASCL2, EPHB2, PHLDA1, SOX9 and OLFM4. Intestinal organoids at P5 were significantly upregulated or similar between intestinal organoids and the intestine.

\section{Supplementary Files}

This is a list of supplementary files associated with this preprint. Click to download.

- S1.png 
- S2.png

- S3.png

- S4.png

Page 20/20 